\title{
A Method to Solve the Communication Gap between Designers and Users
}

\author{
Jeichen Hsieh ${ }^{1}$, Chia-Ching Lin $^{1}$, and Pao-Tai Hsieh ${ }^{2}$ \\ ${ }^{1}$ Department of Industrial Design, Tunghai University, Taiwan, R.O.C. \\ ${ }^{2}$ Tai-Chung Institute of Technology, Department of Department of Commercial Design \\ National, Taiwan, R.O.C. \\ jeichen@thu.edu.tw, linny0709@hotmail.com, hsiehantit.edu.tw
}

\begin{abstract}
There are always discrepancies when abstract design concepts are transferred to solid products. How to make sure that design concepts are conveyed exactly via products? To develop the early stage prototypes for tests and surveys is one of the solutions. The research applies POE (Post-Occupancy Evaluation) on prototypes of students' design cases repeatedly. The result revealed that product prototype POE can anticipate the performances of products in final evaluation as an evaluation can predict post-production consumer reception. It suggests that performances of product prototype by POE would be clarified if extraneous variables are under strict control in advance. Two cases show chaos phenomenon, to probe into the field of students' design activities with grounded theory might help to unearth some discovery.
\end{abstract}

Keywords: Post-Occupancy Evaluation, Prototype, Cognitive Differences.

\section{Introduction}

When the abstract design concepts are transferred into concrete products, there will always be some degree of gap. Crilly et al [1]describes product design as a communication process, the designer message encoded in the product and the customer, it is then decoded and explained by the product; However some cognitive problems will always exist, such as Norman [2] stated: designers and users do not directly communicate through their products and end-user communication only. Therefore, if a designer wants to confirm whether he sent a proper message to the user, one way is through the development of early prototype testing review.

Prototyping and testing, product development into practice in several functions, can be used as the company's communication media. It can help designers to develop ideas and also be used to test the product to verify if the design is appropriate. In the pre-evaluating new product ideas, the designer wishes to know whether the product concepts is a good idea in the eyes of the others, whether the design principles to achieve this goal simply tell them what the product can do and how these different products where the usibility of sketches, precision drawings or mock model can achieve the purpose. If the product is manufactured as a prototype for testing, the reaction may be related to the design and details [3]. In short, in the development of a 
concrete answer after the design program, you need to examine whether it meets the original objectives.

Coughlan and Mashman [4] note that when more than one prototype must be selected or, usually ask the customer to choose the most attractive proposal for further development or improvement of the missing to do, in this single-phase of the assessment the customer does not make the right judgments, easy to provide false information, causing errors on the design decisions, leading to the development of a design or development should not veto the design of risk should not be rejected. Also, ask the customer to select their most attractive proposal is consistent with the original target market or to meet the original design proposal? It needs to verify. By the use of the construction sector assessment of POE (Post-Occupancy Evaluation) method, a designer could understand the intent and the user intent and thereby shorten the gap among multi-application architecture and urban planning and other fields. Follow-up with researchers to apply it to industrial design to view products in the design and planning on the information to know whether the user can really feel the product design and planning on the intention and the assessment can be used in nextgeneration knowledge acquisition products improvement; Since the ex post review of POE can be used as the product improvement policy, whether that is in the prototype stage of development to evaluate and improve the implementation of POE, whether the effects are significant? That is the research focuses.

In education, there are often many steps between the first ideas to the completion of modeling, with teacher's guidance for improvement. This is similar to the work industry, when the design goes through internal assessment decisions by the superiors. However, in the interview with the former Toyota Motor Minister of Design, it is stated that the early decisions made by a superior often result in errors, with no further assessment due to a systematic problem. We can see that the practice has to be effective in practice. POE prototype response to the research, but the development of the field of industrial design prototype POE method, import the repeated amendments to the process of evaluation and assessment of students design prototype, the design concept and the actual performance gap and give recommendations to encourage students to better approximate the design, prototype and then try to understand if the implementation of POE cases can result in significant impact on students.

\section{Literature Review}

\subsection{POE Method}

POE contains research and review the previous assessment of the research prototype, is to grasp the principles and characteristics of POE, to explore its content and evolution, and by what is the prototype, the prototype is to investigate the scope of the establishment.

POE method views the environment, the effectiveness of architectural design and planning, accumulation and sorting through the data as a basis for future improvements. In contrast, POE used in industrial design, should be able to view the product information on the design and planning, and to understand whether the user can really feel the product design and planning with the intention; Liguan Yao in the 
POE-in home appliances refrigerators Case studies, for the first time to understand the use of POE in the product design, users and designers the difference between ideas, products in use after those changes, the user may misuse act and new patterns of behavior. Architecture and industrial design are compared in the different POE, and the establishment of suitable operating model for industrial products and related POE.

The nature and functions of POE have somewhat different views. Sommer defines the POE is the "built environment for the use of a systematic examination of ways to obtain useful information"; Rabinowity says the POE is the "built environment for understanding and improving the effectiveness of a method of using"; domestic scholars have proposed to use assessment as a "specifically refers to the use of the building or building environment research". Meanwhile Shyh-Meng Huang think "POE's point of view the first emphasis on space and activity (or use) whether there is correspondence between the occurrence of conflict issues, the fact that these contradictions to resolve these contradictions." In addition, according to Zimring and Rirenstein, POE is "to access, observation, questionnaires and other methods to understand user views on all aspects of building work", which are more operational definition, the object between people and buildings define. And Zimring's another definition of POE is "diagnostic work on the environment, and this environment is by design for human use after". All in all, the POE can be generalized and treat the efforts of the past as a reflection and decision-making, from seeking to increase understanding and further improvement of the road. More narrow view of architectural design from the perspective of users, such as exploring the response of the built environment to improve the design of future cases similar to the decisionmaking; or to assess the functional performance of buildings, and use and planning objectives and content of the original comparison; or to act with the architects on the use of assumptions compared to understand the differences and to explore the cause during different reasons.

POE and rationalization of a systematic process, emphasizing the position and method of objective evaluation and assessment criteria such as characteristics of importance is the result of its and the "Architectural Review" (Critique) different places. In other words, POE is the use of social science research methods of the built environment and people (especially users) to do the relationship between integrity and depth.

The Presier based on the length of the POE application functionality into three stages:

\section{In the short-range effect on:}

(1) determine the success or failure of the building.

(2) make recommendations on the need to fix the problem.

(3) in the development project budget to provide some information.

2. medium-range effect on:

(1) to help determine the suitability of the building to continue to use, modify or re-build and other measures.

(2) resolve the supply dimension of existing buildings.

3. the effect on the remote:

(1) POE can be based on the findings to the design of future buildings. 
(2) basic information designed to enhance and improve the formulation of assessment criteria and guidelines.

(3) to improve the qualitative aspects of the past performance of the building methods of measurement.

And, Preiser [5] presented in the writings of three levels of POE studies can be used in any form or size of buildings or facilities:

\section{Indicative POE (Indicative POE):}

The main problem is to find that the advantages and disadvantages, success and failure, for short-term assessment. General to file the collection, performance evaluation, field reconnaissance and interviews of four methods required time from 2 to 3 hours to a day or two days.

\section{Investigative POE (Investigative POE):}

Index of POE is that the results of important issues, and investigative POE need to get to the bottom of the topic for a more detailed investigation. The main basis for the formulation of assessment criteria related to literature, and reference to recent cases of similar assessment and therefore the higher its credibility, more objective; need for about 160 to 240 hours.

\section{Diagnostic POE (Diagnostic POE):}

Diagnostic POE is a comprehensive and in-depth assessment, and involves many variables, the use of questionnaires, surveys, observation, the actual measurement and scientific method and other data collection methods, aims to understand the actual environment and facilities, the relationship between behavior and the variables and the relationships between variables; assessment results can be compared and scientific standards, it is quite accurate, and can enhance the knowledge of the building, design guidelines, and even make amendments to laws and regulations; time from months or even a year or more.

\subsection{Industrial Design Prototypes}

Through Science Direct and UMI ProQuest Digital Dissertations search, keyword Evaluation, Product and Industrial Design Prototype door in search results, similar to the original planning POE design information for the control of the ex-post evaluation methods used in the prototype or product literature little.

If Section I, POE than used in the construction field, Liguan Yao used the first time in the refrigerator POE product evaluation, and Cai Shijie dehumidifier product innovation and POE. The context of the POE is no more in-depth understanding of, for example: There is no understanding of POE to assess which building projects? How these projects generate? In addition, POE is not described how the three levels used in the field of industrial design. Coughlan and Mashman [4] create the prototype of a single-stage (single-session) of the assessment, the customer does not make the right judgments, the easy to provide false information, causing errors on the design decisions, it advocates with more than one assessment, which is to allow customers to look at a few prototypes, to reduce "side of the edge," Evaluation of the potential to make the crisis, and concluded that: the assessment of pre-production market should 
be able to predict customer acceptance of the situation after ; cultural context is often in the aesthetic aspects of the product play an important role and the success of aftermarket, and the manufacturer's image and reputation, people use their past experience in related products and functions, etc., will affect the judgments of the product, so In considering a potential product, the other for the assessment can not be ignored. Further, Crilly et al [1] summed products are aesthetic, symbolic and practical functions such as 3 large, and suggested that further research is to develop appropriate ways to examine all aspects of product performance, and even to predict whether the product can stimulate the customers as expected reaction; Heufler [6] also indicated that the works of aesthetic products, symbolic and practical basic functions such as 3 large, we can see three basic functions of large argument so far is more consistent. Based on the above, Coughlan and Mashman said the current product design, prototype for the evaluation of other still room for development, and evaluation of pre-production and after-market customer response related to sex. Liguan Yao pointed out that the POE applied to assess the feasibility of industrial products, and products, POE survey that can be assessed and revised from generation to generation products; If POE used in the prototype evaluation, and POE function of feedback and feed forward echoes; use of the prototype POE assessment feedback and make improvements, but the effect is the role of feed forward in the product (eg, Coughlan and Mashman said above the predictive effect), it may form a better assessment of the prototype approach. Baxter [3] that the design process and models used in the prototype can be divided into a structural characterization of the model with functional characterization of the prototype and the characterization of both structure and function of the prototype.

\subsection{Research Questions}

POE in the construction sector or field of industrial design applied to the POE, and irrespective of whether the POE in the field of industrial design, whether the spirit of the original POE, POE common research approach is used, the original planning and user cognitive differences between it reported; product assessment is an established fact, the conclusion to provide improved directions, did not address the design intent and user POE reduced cognitive performance.

The product design process, designers are thinking how to be sure users understand which way is through the development review of the early prototype testing to verify design concepts to convey the relevance of the conversion, in addition to the appropriate research approach to solve, but also To know how it works.

The focus of research is to expose "POE users to shorten the design intent and cognitive differences," the performance of the POE method is applied to the first prototype; use of prototyping and testing, repeated evaluation and correction (Assess $\&$ Refine) process to implement the product prototype POE, to answer whether the use of prototype POE, the subjects in the final assessment of whether a significant difference? And whether subjects were able to predict the final response? Such as preproduction evaluation, it can predict after-market customer acceptance of the situation. 


\section{Methodology}

Assessment of the prototype is done by the students of the Department of Industrial Design, Tunghai University. Including: (1) chair prototype POE, sophomore design; (2) acrylic and re-design after POE, junior design; (3) cross step onto the track of the next semester, the experimental group and control group were two senior project students. Figure 3.1 shows the order of the processes. First, to understand the feasibility of POE applied to prototype, then the chair directly to the sophomore design prototypes for evaluation, and then go back and search for literature, will be integrated and field data to construct the theory is not only the documentation from the field come, and studied literature in the field of temporary and verified, so the POE literature review in the chair after the end of the prototype, and has practical experience with the designer to understand how the industry's implementation of the prototype assessments.

After the prototype chair determine the feasibility of POE, but also learn from the literature review, should be followed in the construction sector POE way to the development of industrial design product prototypes POE approaches, then submitted documentation inductive method POE model prototype construction; time study gradually reduce the focus on design intent of POE and the user perceived performance, the use of the student work on is an attempt to POE will review the information back to students, encourage students to better approximate the design.

POE prototype is used to understanding other sides of the implementation of multiproduct (POE prototype chair facing a single product do not), so in the third year of its implementation of the prototype acrylic and submit works of students attended a common problem, according to procedures to conduct the first POE (cancellation), and re-design of the second post-POE. Also at the same time senior graduation project, the first experimental design in order to grasp the upper and lower across the time course of the semester, the whole experiment is divided into five senior mileage, and established to operate the variables and experimental and control group arrangements, senior experimental design.

After the experiment the first two milestones, the last semester of study will come to an end, then has to do the assessment work done to review and feedback to the prototype POE approaches, after continue next semester to complete three assessments.

\section{Results}

Implementation of POE from the understanding of the feasibility of the prototype began with repeated cases of students to design prototype implementation of POE, there are findings as following:

POE prototype will be used in cases of students work, repeated evaluation and improvement of its prototype implementation of the recommendations in the final evaluation of subjects. From the Scheffe method of post hoc comparison, the experimental group and control group work did not reach 0.05 level of significance. Hence there are differences between the performance but not significantly; and then ranked the performance seems to work, the first born of the experimental group B 
single rinsing mug, the second born of the control group D with a group of cold tea, which means that the work done using POE Performance is not necessarily better, and that work without POE is not relatively to poor performance. There is no significant effect in the POE performance of this four students.

Works in the experimental group and the final assessment four miles, respectively, by the independent sample t test and Scheffe method of post hoc comparison, did not reach 0.05 level of significance, the results indicated that the two assessments showed no significant difference. In other words, four miles from the school final results of the evaluation will be able to get a glimpse of a new generation of development works in the school's performance, it can be expected to judge cases of POE prototype high school students work, the performance of the final results of the assessment; such as the assessment of pre-production, to predict product Listed after the customer accepts the situation.

POE on the prototype works in the of secondary school students showed no significant effect, said the POE literature review the research work carried out under natural conditions, rather than in a controlled experimental conditions, but the process of gradually found it difficult to external variables do better isolated defects, such as designers to reverse the verdict, and the design capabilities of different items, etc.; meaning POE used in the prototype will need to be in a better controlled experimental conditions, if asked to set up product items and designers can not overturn and other control measures, should also help clarify the effectiveness of POE prototype. And when items are not at the same time, it is more difficult to clarify the diagnosis POE prototype design and prototype feedback on the effectiveness of what happened, as the case of the pot with the robot lamp, table lamp itself, the robot may be difficult to obtain subjects who recognition, but if the two table lamps designed by key design robot, the more consistent base of comparison. Senior test mileage II, Kathy Chang, single rinsing mug after assessment found that there are functional, and that cup diameters which are too small will cause the brush bristles into the cup on the face and prevent lack of water movement, the feedback survey after the diagnosis of POE Mileage three functional assessment found that cup to drink water on the bristles have not obstructing movement. As Norman [2] described, recursive testing and re-design, the level of behavior (functional) design has played a good role, but not necessarily suitable for instinct (aesthetic features) or reflection level (symbolic function) design.

Some recommendations are as following:

From the works of experimental and control groups, there is no significant difference in the performance of views. The case did not present a significant benefit by students, but the diagnostic feedback POE prototype is more significant for a senior designer to reflect on their instinct.

Prototype POE recommends using only the investigation of the two indicator levels, as indicators for short-term assessment of the POE. The main problem is found that the advantages and disadvantages, and success and failure, the time required from 2 to 3 hours to a day or two days, the study's assessment of the mileage are all investigative POE found in the indicator problem, its more detailed investigation are applied to the assessment of all POE takes about two weeks. The POE is the diagnostic variables and variables related to the relationship between the time required from months to a year or more [5], the study did not use the highest level which is necessary to implement POE. It seems too difficult to match the time used in 
the prototype, because the results are too late to feed back to designers in product development, unless a considerable length of time.

1. In this will complete prototype development of skills needed for POE (POE after 30 years of architectural development), the main results of the current prototype in the proposed POE approaches, but more specific items such as: functional aspects of environmental protection, a symbol of the functional aspects such as cultural and social context detection method, and using better ways to detect, follow-up study to be itemized and specializes in the integration of knowledge into prototypes and then POE research approach.

2. Research in the "sub-blocks method of investigation" did not get the better of, because you can more easily partition the building blocks, but the product of a partition block would probably lose its significance (based on gestalt psychology) and would be difficult to define the scope. For example: a robotic desk lamp where block $\mathrm{A}$ is defined as the palm of your hand to the shoulder, but why not from the palm to the elbow? There seems to be no sufficient reason to support such a regional division, the impact, for example: only know that the poor performance of the prototype and beautiful, but not that "the beauty of which part of the poor"; raises a question: how to detect the aesthetic (or other) part of a negative evaluation of the exact place? Can only be subjects for the prototype general awareness of the negative evaluation of the qualitative data records, if the designer knows exactly which part or range of subjects is not understood and difficult to clarify. Suggest that future developments need to improve that part of the prototype approach.

3. The industry does not face the fear of using more innovative design of the phenomenon in Taiwan. By POE and Decision Sciences, it might help the evaluation of making prototypes and let the designers be more convincing.

4. From the chair prototype POE we found that the general assessment of the personnel evaluation of subjects and the scores for teachers were higher than the assessment of staff; a glimpse into different plays (Occupy) and occupies the length of time seems to influence the form of assessment, like business involvement in the field of the impact of different decisions on the purchase, so the prototype POE Marketing and Business Studies (Marketing) areas should be able to do more closely with the possession of such different forms, such as: a packed, unpacked and made available any consumer touch, but packaged and exposed part of the product design without instructions, etc., the impact on purchasing decisions.

5. In the assessment of association via a qualitative record of items to find work similar appearance can lead to more in-depth articles of association or emotional detachment, such as: single rinsing mug reminiscent of a partner's time. Since the emotional detachment can sometimes change or advertised products through methods such as enhanced, thereby allowing the consumers feel are "their" products [6]. To use POE prototype to explore the use of items and do not use items in advertising effectiveness or the impact on consumer purchasing decisions could be considered.

6. The current assessment is more artificial data interpretation, diagnosis and recommendations vary susceptible to assess product prototypes more cumulative. It is expected to organize the analysis and diagnosis of the rules, such as: with correlation analysis, can determine why the performance of the project or sort out the rules for construction of expert system. 
7. POE survey is to assess and generation of products and buildings, but no studies have product or building to be followed and diagnosis. It does not show the generation better or not. In terms of design activities for students, graduates of the next track are to follow on the topic if the term by the POE diagnosis, whether the performance of a new generation of work can be better will be explored.

8. Cases, a glimpse into the Chaos of the phenomenon, are beyond the scope of the study. Scholars in different fields have been found to chaotic phenomena, the proposed follow-up study in this direction may be able to open another field of industrial design a "nonlinear" perspective. For example: Grounded Theory approach taken directly to the students in the fields of design activities and to observe the chaotic phenomena and create some new concept and theory.

\section{References}

1. Crilly, N., Moultrie, J., Clarkson, P.J.: Seeing Things: Consumer Response to the Visual Domain in Product Design. Design Studies 25(6), 547-577 (2004)

2. Norman, D.A.: Emotional Design (Emotional Design: Why We Love (or Hate) Everyday Things) (Que Lan Weng. Garden City Culture Limited, New York (2005)

3. Baxter, M.: Product Design and Development (Product Design: A Practical Guide to Systematic Methods of New Product Development). Kuni Press, New York (1998)

4. Coughlan, P., Mashman, R.: Once is Not Enough: Repeated Exposure to and Aesthetic Evaluation of an Automobile Design Prototype. Design Studies 20(6), 553-563 (1999)

5. Preiser, W.F.E., Rabinowitz, H.Z., White, E.T.: Post-Occupancy Evaluation. Van Nostrand Reinhold Company, New York (1988)

6. Heufler, G.: Design Principles: From Concept to Product Formation (Design Basics: From Ideas to Products). Longxi Books, New York (2005) 